\title{
Genetic study of transthyretin amyloid neuropathies: carrier risks among French and Portuguese families
}

\author{
V Planté-Bordeneuve, J Carayol, A Ferreira, D Adams, F Clerget-Darpoux, M Misrahi, G Said, \\ C Bonaîti-Pellié
}

\section{INTRODUCTION}

Among the hereditary systemic amyloidosis, transthyretin (TTR) neuropathies (OMIM \#176300) are devastating disorders with an autosomal dominant transmission, expressed mainly as a progressive fibre length dependent sensorimotor polyneuropathy and life threatening autonomic dysfunction. ${ }^{1}$ Initially, the condition was recognised in northern Portugal, in the area of Povoa de Varzim, where the prevalence has been estimated at $1 / 1000 .^{23}$ Other important clusters of families were subsequently reported in Japan, initially in two limited geographic areas (the districts of Arao and Ogawa), ${ }^{4}$ and in the north of Sweden. ${ }^{5}$ The condition is now known throughout the world, including various European countries. ${ }^{6}$ Among the 75 pathogenic TTR variants identified so far, Val30Met is the most frequent, and is virtually the only one described in Portugal and Sweden. ${ }^{78}$ By contrast, in Japan, the TTR point mutations are highly heterogeneous, with more than 20 different substitutions, including Val30Met, described. ${ }^{9}$ In France, a previous study found a similar heterogeneity. ${ }^{10}$ Depending on the geographic origin, the mean age of onset ranges from 30 to 70 years. A variable clinical expression is also observed, with restrictive cardiomyopathy, carpal tunnel syndrome, and vitreous opacities often encountered, whereas renal involvement is less common. These manifestations, along with the discrepancy in age of onset, account for phenotypic variations. However, virtually no genotype-phenotype correlation seems to exist. To supply the main source of mutant TTR, liver transplantation has been proposed as a treatment for the disease for the last 10 years, with promising results. In the Val30Met patients, it virtually suppresses the production of the mutant TTR in the serum and slows the progression of the neurological symptoms that otherwise have a fatal outcome in less than 10 years. ${ }^{11}$ Another important aspect of the management of affected families is the recent availability of predictive molecular genetic diagnosis. Before the availability of liver transplantation, the presymptomatic or prenatal testing in relatives of patients raised many ethical issues. Nowadays, it is more widely performed in the frame of genetic counselling. Estimation of the risk for mutation carriers of being affected is essential. In order to support the choice of the family members, precise information is required on the clinical and genetic epidemiological background of the condition in the different geographical areas. After the historical study of Andrade, ${ }^{2}$ which concluded that inheritance is dominant with very high penetrance, some genealogical studies suggested that penetrance was probably incomplete and variable among families. ${ }^{32-16}$ To gain more insight into the subject and to obtain penetrance estimates necessary for both genetic counselling and a better understanding of underlying mechanisms, and to test for possible differences between individuals, we performed a genetic study of TTR amyloid neuropathy in two separate populations, of French and Portuguese origins respectively.

\section{Key points}

- Transthyretin amyloid neuropathies are devastating autosomal dominant conditions, first described in northern Portugal, and now recognised across the world. Depending on the geographic origin, wide variations in age of onset, sex ratio, and clinical presentation account for the phenotypic spectrum of the disease.

- Previous genealogical studies have suggested that penetrance is incomplete and variable, but this parameter has never been estimated accurately. We performed a genetic study of transthyretin amyloid neuropathies in two separate European populations.

- A method of estimation of penetrance was developed based on maximum likelihood using a survival analysis approach. Sixty two unrelated index cases of French origin and 48 of Portuguese ancestry entered the study.

- Genealogical investigations, clinical information on symptomatic cases, and results of the genotyping of voluntary family members were obtained in relatives of 46 French and 33 Portuguese kindreds, respectively.

- Up to 12 different pathogenic transthyretin variants were detected in the French kindreds, including Val30Met, whereas Val30Met was the only variant in the Portuguese. The penetrance curves were found to be quite different between the two populations, with an age of onset much lower in Portuguese than in French index cases (mean (SD) 34 (10.5) v 58.9 (12.3) years), and a penetrance by the age of 80 years that was slightly higher in Portuguese $(91 \%$ v $86 \%$ ) than in French cases. Surprisingly, the risks were found to be lower in the French Val30Met carriers than in carriers of other mutations $(p<0.02)$.

- Our results provide an important input for the management of asymptomatic carriers in the Portuguese and French families. They also contribute to a better understanding of the mechanisms underlying the disease expression.

Abbreviations: $T R R$, transthyretin 
MATERIALS AND METHODS

Diagnostic criteria for index patients and symptomatic individuals

A TTR amyloid neuropathy is considered in patients who manifest a subacute or chronic progressive sensorimotor and autonomic axonal polyneuropathy. Until 1991, the diagnosis was based on: (a) the presence of TTR positive amyloid deposits on one or more of the biopsied tissues, namely sensory nerve, accessory salivary glands, skin, periumbilical fat, or rectum; and $(b)$ a documented family history of TTR related amyloidosis. Since 1992, the availability of molecular testing has allowed the diagnosis of TTR in apparently misleading cases, especially in those with sporadic presentation. Since then, in our experience, identification of a pathogenic TTR variant by DNA analysis has become the only diagnostic test. In the present study, index cases were all unrelated patients, diagnosed with TTR related amyloidosis, and investigated in the neurology department of Bicêtre Hospital.

\section{Genealogical investigations and recording of clinical information}

A genealogical enquiry was carried out in every family by two investigators (AF and VPB) and data entered were using Cyrillic software (version 2.0). In non Portuguese cases, the French origin of the family was traced in at least two generations above the index patient, using data from public registers and with the help of the index cases and their relatives. Information on first, second, and third degree relatives was recorded, including the date and place of birth and, if appropriate, the date and cause of death. For all symptomatic living individuals, the diagnosis relied on the criteria defined above. A detailed history of the disease was taken from the patient and/or completed with information from medical records. In particular, the date of onset of the disease was assessed as accurately as possible. Carpal tunnel syndrome was not taken as the first manifestation because of its high frequency in the general population.

In the majority of deceased patients, the same diagnostic criteria were used, based on information obtained from the family members, completed with the medical sources. In a few more distantly related deceased individuals, the diagnosis relied solely on family reports. Such cases were included in the study only if the clinical description was highly suggestive of the diagnosis.

\section{Genotyping of family members}

In the French kindreds, the probands were asked to contact their relatives aged over 15 years, and to propose that they participate in the study, the purpose of which was explained through a written document. Every subject willing to participate had to contact one of the two investigators. A visit was then organised to record relevant medical information and to allow a physical examination. At the same time, a blood sample was obtained for DNA analysis from individuals aged 18 years or older, after informed written consent. The genotyping data were confidential, but the sampled subjects were allowed to know their genetic status if they wished, under strict conditions approved by the local ethic committee. The genetic counselling protocol was similar to the one used for presymptomatic diagnosis in other neurodegenerative disorders, such as Huntington's disease.

The same procedure could not be applied in the Portuguese families. Consequently, genotyping was not systematically offered to the family members. In some cases, it was determined as part of predictive diagnosis, along with a clinical evaluation and genetic counselling in the neurogenetics clinics. In other cases, patients' relatives were aware of their genetic status because of a molecular diagnosis performed elsewhere. In such cases, reliable clinical information was recalled from the individuals and their medical records.

\section{DNA analysis}

Molecular genetic analyses were carried out. Briefly, after extraction of genomic DNA from the peripheral blood lymphocytes, PCR amplification of the four exons of the TTR gene was performed, using oligonucleotides previously described. ${ }^{6}{ }^{17}$ Direct genomic sequencing of both sense and antisense strands of the amplified products was performed using the Taq Dideoxyterminator Cycle Sequencing kit and the 373A sequencer (both Applied Biosystems, Foster City, CA, USA). Digestion with the restriction enzymes BsII or NdeI was performed on the $415 \mathrm{bp}$ PCR product of exon 2 to confirm the two mutant alleles newly identified in this study.

\section{Estimation of penetrance}

\section{Statistical method}

To provide valid estimates of penetrance, it was necessary to adjust for ascertainment bias, as families were selected through affected individuals. Therefore, a method of estimation was developed, based on maximum likelihood using a survival analysis approach, and adapted from ARCAD, 18 which estimates penetrance using phenotypic information from all individuals, even the untested ones (that is, those with unknown genotypes), and corrects for selection bias. This method has also the advantage of allowing for an overrepresentation of affected individuals among those who are tested, and has been successfully applied to the estimation of cancer risks in $p 53$ mutation carriers. ${ }^{20}$

As the original ARCAD method was substantially modified for the present estimation, we provide here a complete description of the method used in this paper.

$L_{f}$ is the conditional likelihood of a family $f$,

$$
L_{f}=\frac{N_{f}}{D_{f}}
$$

where $N_{f}$ is the contribution of all members of family $f$ to the likelihood corrected by $D_{f}$ for ascertainment. $N_{f}$ may be written as follows:

$$
N_{f}=\sum_{w=1}^{\Omega} \prod_{m=1}^{n} P\left(\text { geno }_{m, w}\right) \cdot P\left(\text { pheno } \text { geno }_{m, w}\right)
$$

where $\Omega$ is the number of all possible genotypic configurations, depending on the number of untested individuals, and $n$ is the number of individuals of family $f . P\left(g^{2} n o_{m, w}\right)$ is the probability of genotype of individual $m$ within configuration $w$, and $P($ pheno/geno $m, w)$ is the probability of the phenotype of this individual, given his/her genotype within configuration $w$.

Given a genotype, what is the probability of a particular phenotype? If $F(t)$ is the penetrance function for carriers (the penetrance is assumed to be 0 in non-carriers), the contribution to the likelihood of an unaffected carrier $m$ at age $t_{m}$ is

$$
\text { Pphen }_{m}=1-F\left(t_{m}\right),
$$

that is, the probability that individual $m$ will still be unaffected at age $t_{m}$ (survival probability), and the contribution to the likelihood of an affected individual is

$$
\text { Pphen }_{m}=F\left(t_{m}+1\right)-F\left(t_{m}\right) \text {, }
$$

that is, the probability of being affected at an age of onset included in the 1 year interval

$$
\left[t_{m} ; t_{m}+1[\text {. }\right.
$$


To take into account the possibility that some carriers will never develop the disease, we introduced a parameter $\kappa$, the fraction of individuals that would never be affected. ${ }^{21}$ The probability $l-F\left(t_{m}\right)$ that individual $m$ would still be unaffected at age $t_{m}$ is

$$
l-F\left(t_{m}\right)=\kappa+(1-\kappa)\left(1-F u\left(t_{m}\right)\right)
$$

where $F u\left(t_{m}\right)$ is a parametric function describing the cumulative probability of being affected. We chose the Weibull model with parameters $\lambda$ (scale parameter) and $a$ (shape parameter) as the parametric function, because it is widely used in parametric risk estimation and because of its flexibility to adjust to observed data. This then gives us:

$$
F u\left(t_{m}\right)=1-\exp \left(-\lambda\left(\left(t_{m}\right)^{a}\right) .\right.
$$

What is the probability of genotypes of individuals in the families?

If $i$ is the genotypic status, ( $i=0$ for a non-carrier and 1 for a carrier), and $p_{i}$ is the probability of having genotype $i$, an individual m contributes to $N_{f}$ by:

$$
p_{1} . \text { Pphen }_{m, 1}
$$

if $m$ is a carrier,

$$
p_{0} \cdot \text { Pphen }_{m, 0}
$$

if $m$ is not a carrier

$$
p_{0} . \text { Pphen }_{m, 0}+p_{0} . \text { Pphen }_{m, 1}
$$

if genotype of $m$ is unknown, where $p_{i}$ is expressed as a function of the frequency of the mutated allele in the general population, assuming Hardy-Weinberg equilibrium for a founder (parents' status unknown). Otherwise, this probability depends on parental genotypes, assuming Mendelian transmission. In the analysis, disease allele frequency was assumed to be very low and fixed to the value 0.001 , after checking that estimates of penetrance were not sensitive to the gene frequency value. Regarding untested individuals, the probability of their genotype depends not only on their parents, but also on their offspring's genotypes. Indeed, if at least one of the children of an untested individual is known to be a non-carrier, taking this information into account modifies the probability of this individual of being a carrier. To use all the available information provided by family members, we used the method adapted from Elston and Stewart. ${ }^{22}$

The ascertainment correction, $D_{f}$, is the probability that, in familiy $f$, there is at least one proband (one affected individual) belonging to the sample, which is equivalent to:

$$
D_{f}=\sum_{w=1}^{\Omega} \prod_{m=1}^{n} P\left(\text { geno }_{m, w}\right) \cdot \text { Pprob }_{w}
$$

where $\operatorname{Pprob}_{w}$ is the probability that there is a least one proband in configuration $w$.

If $\pi$ is the probability of ascertainment, that is, the probability that an affected individual is a proband, this probability may be written as:

$$
\text { Pprob }_{w}=1-\prod_{m=1}^{n}\left[\left(1-F\left(t_{m}\right)\right)+F\left(t_{m}\right)(1-\pi)\right]
$$

The probability $\pi$ is usually estimated from the distribution of probands among affected family members, assuming that affected individuals are independently ascertained. ${ }^{23}$ As the hypothesis of independence of ascertainments in the same family is not tenable in the present study, the parameter $\pi$ was set to various values to evaluate the sensitivity of penetrance estimates to its specification.

Finally, the likelihood for the whole sample of families is the product of likelihoods over all families.

A program has been written that uses the maximisation procedure GEMINI as a subroutine, ${ }^{24}$ and provides maximum likelihood estimates of the parameters $\kappa, \lambda$, and $a$. Confidence intervals of penetrance at a given age were obtained by applying the delta method. ${ }^{25} 26$

To test for a difference according to gender, we used a likelihood ratio test (LRT). This test compares the maximum likelihood value $L_{1}$ obtained assuming the same penetrance in males and females to the maximum likelihood value $L_{2}$ obtained when allowing for a difference according to gender. The statistic used, $-2 \operatorname{Ln}\left[L_{1} / L_{2}\right]$, follows a $\chi^{2}$ distribution with a number of degrees of freedom equal to the difference between the number of parameters estimated in $L_{1}$ and $L_{2}$ respectively. The same principle was applied to test for a difference in penetrance according to the mutation involved in the French group and to the origin of the group (French or Portuguese).

\section{Family data used}

All family data providing information on the risk of being affected for a relative were retained. Thus, individuals at risk or patients, with reliable information on their date of birth, their age of onset, or their date or age of death, if adapted, were included. In a minority of affected cases, the age of onset was extrapolated from the age of death, using the mean duration of the disease calculated respectively for the sample of French and Portuguese index patients. The branches of the families without information on the individual's phenotype or date of birth, or on the age at first manifestations were excluded. On the basis of the known age of onset in various populations of TTR amyloid neuropathy, individuals younger than 16 years were not considered in the present study.

\section{RESULTS}

\section{Subject demographics}

Between 1988 and 2001, 110 unrelated index cases were referred to our department fulfilling the diagnostic criteria of TTR hereditary amyloid polyneuropathy. Among them, 62 were of French origin and 48 of Portuguese ancestry. No French family was found to have any Portuguese ancestor.

Among the French index cases, five patients were deceased, three could not be reached and eight were unwilling to participate. Among the referred Portuguese index cases, four were deceased, postal address was unreliable for 10 and one refused to participate in the study. Genealogical data were thus collected from 46 French and 33 Portuguese families.

\section{TTR variants}

Val30Met was the only detected heterozygous substitution in the Portuguese kindreds. In contrast, up to 12 different pathogenic TTR variants were detected in the French kindreds, including Val30Met and six newly detected mutations. ${ }^{6} 1027$ The substitutions were all present in the heterozygous state and distributed as follows: Val30Met (27 families), Ser77Tyr (11), Ser77Phe (6), Thr49Ala (4), Ilel07Val (5), Tyrl16Ser (2), Ala91Ser (2), Val32Gly (1), Lys35Asn (1), Phe44Tyr (1), Ser50Arg (1) and Glu61Lys (1). The previously unreported T1686G and T1722A missense point mutations in exon 2 of the TTR gene predicted Val32Gly and Phe44Tyr substitutions. These two unreported point mutations create a restriction site for the endonucleases BslI and NdeI, respectively. Restriction analysis of the PCR product 
of exon 2 confirmed that the affected individuals were heterozygous for one mutant allele in these families (data not shown). In contrast, the additional BslI or NdeI sites were not found in 100 healthy unrelated controls, indicating that both mutations are unlikely to be polymorphisms. The French families in which family data could be obtained included 20 Val30Met families and 26 kindreds carrying other variants.

\section{Isolated and familial cases}

In 20 French families, the proband was the only affected individual of the family $(43.5 \%)$. In the remaining 26 kindreds $(56.5 \%)$, there were 2-14 affected members spanning 1-4 generations. Twenty nine Portuguese index cases $(87.8 \%)$ had a positive family history of TTR amyloid neuropathy, and four patients presented as isolated cases.

\section{Age of onset, sex ratio, and disease duration}

The 62 French index cases included 44 men and 18 women, averaging 58.9 (12.3) years (range 16-78), with no difference between males and females. In this sample, $85.5 \%$ of the patients had their first symptoms after the age of 50 years. A minority of the patients $(6.5 \%)$ were symptomatic before the age of 35 years (fig 1). Among the Portuguese cases, there were 27 men and 21 women. The average age of onset was 34 (10.5) years (range 22-68), with no gender difference. Most of the patients $(75 \%)$ presented with initial symptoms before 35 years of age, and only five of them became symptomatic after 50 years of age (fig 1). The difference between French and Portuguese patients for age of onset was highly significant $\left(\mathrm{p}<10^{-10}\right)$. The same difference was found between French and Portuguese affected relatives, with respective mean ages of onset of 50.7 years and 30.7 years $\left(\mathrm{p}<10^{-10}\right)$.

We investigated whether age of onset correlated within families. As the number of affected relatives in families was variable, we could not use a classical correlation coefficient. Instead, we stratified the families according to age of onset of the index cases, setting the cutoff point to the median value among index cases for distinguishing late and early onset families separately in French and Portuguese families. In French families, the cutoff point was 60 years, so the index cases were considered early onset when their age of onset was less than 60 years, and late onset when it was at least 60 years. The mean (SD) age of onset of affected relatives whose age of onset was accurately known was 44.4 (13.8) years in families of early onset, and 58.5 (13.3) years in families of late onset index cases $(p<0.001)$. The same difference was found in Portuguese families. The cutoff point

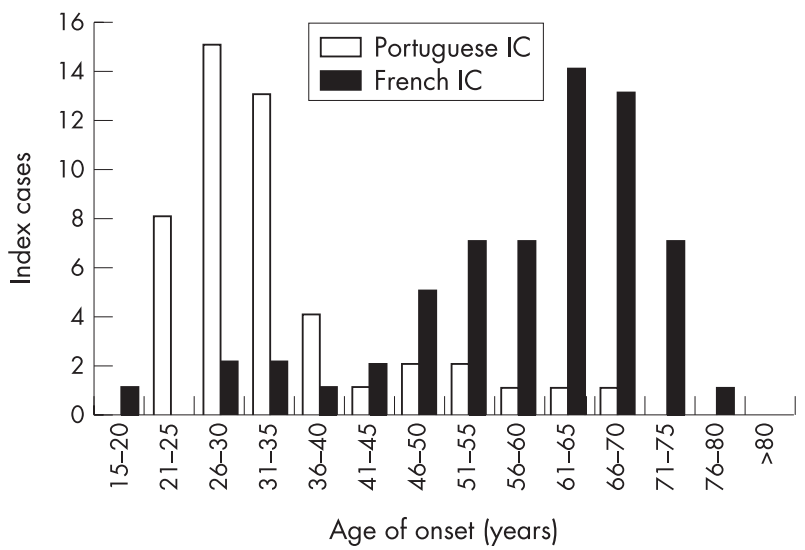

Figure 1 Distribution of age of onset in the Portuguese and French index cases (IC). was found to be 32 years. The mean age of onset of affected relatives was 29.0 (5.3) years in families of early onset and 33.1 (9.1) years in families of late onset index cases $(\mathrm{p}<0.02)$. These differences demonstrate a high degree of correlation within families for age of onset.

There was a significant excess of males among index cases in the French sample, with a sex ratio of $2.4(\mathrm{p}<0.01)$, but this excess was not found among affected relatives, with 38 males and 31 females, respectively. There was no excess of affected males in the Portuguese sample in index cases (sex ratio 1.3) or in affected relatives (sex ratio 1.1).

The disease duration was calculated from the index case in case of death, or by selecting the deceased affected relative who was most closely linked in terms of kinship and birth date. Due to the natural history of the condition, these data were ascertained only in non-transplanted patients. In the French sample, the average (SD) duration of the disease was 8.1 (3.6) years in 35 patients (range 2-18). In the Portuguese sample, the average disease duration calculated from 28 patients was 12.4 (3.3) years (range 7-21).

\section{Investigation of family members}

In the French families, 625 subjects including index patients were recorded, and a DNA sample was obtained in 258 of these family members. In the Portuguese kindred, 384 subjects were recorded, and a DNA sample collected in 139. Table 1 indicates the number of individuals included in each group, according to their phenotypic and genotypic status. In both populations, we obtained clinical information on 242 affected individuals and extrapolated the age of onset in 40 of these for penetrance estimation.

\section{Estimation of penetrance}

Fig 2 shows the penetrance curves in the French sample both as a whole and separately for Val30Met carriers and carriers of other mutations, and in the Portuguese sample, assuming a probability of ascertainment of 1 . Table 2 shows the point estimates of penetrance and the confidence intervals at various ages for these samples. In the French sample, the disease risk remains low (under 0.15) until 45 years of age, and then increases sharply to over 0.8 at 80 years, and no plateau was observed ( $\kappa$ converged to its bound 0 so that only the two parameters $\lambda$ and $a$ were estimated). In the Portuguese sample, the risk increases rapidly from about 15 to 50 years of age and then reaches a plateau at 62 years, with a risk of 0.91 by the age of 80 years. The penetrance function was found to be highly different in the Portuguese and in the French samples $\left(\chi^{2}=155.63,3 \mathrm{df}, \mathrm{p}<0.001\right)$, the risks being much higher at all ages until 75 years of age in the Portuguese. When considering separately the carriers of the Val30Met mutation and the carriers of the other mutations in the French sample, the risks were found surprisingly to be lower for Val30Met carriers $\left(\chi^{2}=7.94,2 \mathrm{df}\right.$, $\mathrm{p}<0.02$ ). When comparing the French to the Portuguese Val30Met carriers, the difference was highly significant $\left(\chi^{2}=127.96,3 \mathrm{df}, \mathrm{p}<0.001\right)$.

When allowing for a difference according to gender, we obtained the penetrance estimates shown in fig 3 and table 3. In the French sample, the penetrance function in males was found to be slightly above the penetrance in females, but the difference was not significant $\left(\chi^{2}=3.33,2 \mathrm{df}\right)$. In the Portuguese sample, the difference was significant $\left(\chi^{2}=\right.$ $13.22,3 \mathrm{df}, \mathrm{p}<0.01)$, with a higher risk in males at young ages, but an age plateau that was reached sooner in life by females than males. When we considered the Val30Met carriers and the carriers of the other mutations in the French sample separately, the difference according to gender was significant in the Val30Met group $\left(\chi^{2}=6.30,2 \mathrm{df}, \mathrm{p}<0.05\right)$ and not in the other group $\left(\chi^{2}=0.08,2 \mathrm{df}\right)$. Thus, in all the 
Table 1 Results of family investigations in the 46 French and 33 Portuguese kindreds

\begin{tabular}{|c|c|c|c|c|c|c|}
\hline \multirow[b]{2}{*}{$\begin{array}{l}\text { Number of } \\
\text { subjects }\end{array}$} & \multicolumn{3}{|l|}{ Genotyped } & \multicolumn{3}{|c|}{ Non genotyped } \\
\hline & Non-carriers & $\begin{array}{l}\text { Asymptomatic } \\
\text { carriers }\end{array}$ & Affected & $\begin{array}{l}\text { Obligate } \\
\text { carriers }\end{array}$ & Unaffected & Affected \\
\hline \multicolumn{7}{|c|}{ French sample } \\
\hline Val30Met & 61 & 44 & 30 & 3 & 179 & 24 \\
\hline Other TTR & 54 & 34 & 35 & 2 & 130 & 29 \\
\hline $\begin{array}{l}\text { Total } \\
\text { Portuguese }\end{array}$ & 115 & 78 & 65 & 5 & 309 & 53 \\
\hline $\begin{array}{l}\text { sample } \\
\text { Val30Met }\end{array}$ & 46 & 24 & 69 & 3 & 187 & 55 \\
\hline
\end{tabular}

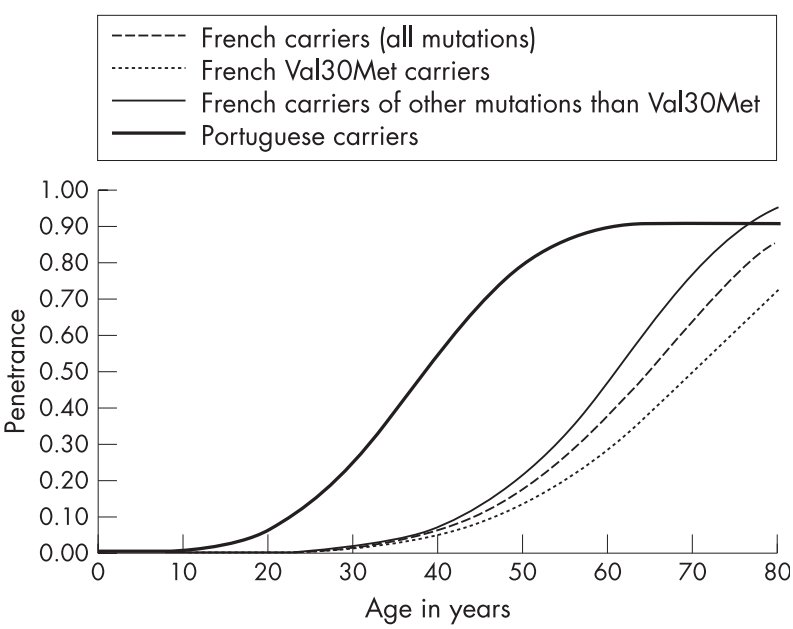

Figure 2 Estimated penetrance function according to type of mutation and geographic origin.

Val30Met carriers, both French and Portuguese, a significant difference in penetrance was found between men and women.

The results were quite similar when allowing for incomplete ascertainment. In the French sample, with a very small value of $\pi$, say 0.1 , the penetrance at 80 years was estimated to be 0.81 , while with a bigger value of $\pi=1$, it was 0.86 , and so remained in the confidence interval (0.81-0.91). In the Portuguese sample, the penetrance was estimated to be 0.88 instead of 0.91 , and so also remained in the confidence interval (0.86-0.95). In general, all the penetrance estimates decrease when the probability of ascertainment decreases, but the differences found for complete ascertainment remained valid when ascertainment was incomplete (data not shown). The difference in the penetrance function remained highly significant between French and Portuguese families, even in the extreme situation of highly different ascertainment probabilities in the two populations, for example, $\pi=1$ for the French sample and $\pi=0.1$ for the Portuguese sample $\left(\chi^{2}=133.14 ., 3 \mathrm{df}, \mathrm{p}<0.001\right)$.

\section{DISCUSSION}

The present study was conducted in two distinct European populations (Portuguese and French) of TTR amyloid neuropathies, which displayed notable genotypic and phenotypic differences. It allowed for the first time an estimation of the risk for mutation carriers of being affected, using molecular data in families and a method adapted to the ascertainment process. Such data are now essential for genetic counselling and management of patients.

The reliability of data, and in particular the accuracy of diagnosis, is obviously an important issue in the estimation of penetrance. In this work, the index patients were all seen in our department, and the diagnosis was confirmed in the majority of affected cases by the presence of amyloid deposits staining for TTR antibodies in biopsied tissues, and/or the identification of the TTR variant by molecular analysis. The diagnosis relied on family reports with no supporting medical records in less than $25 \%$ of affected relatives. The clinical picture of TTR amyloid neuropathy is quite distinct, leading to severe disability in about 10 years, and we do not expect an important rate of error in these familial cases.

Considering genotypes, the Portuguese families had the TTR Val30Met as the only variant whereas the French families were highly heterogeneous, with 12 different pathogenic variants including the Val30Met in 27 families (43.5\%) and six newly identified substitutions. ${ }^{1027}$ The various ethnic origins of the population could explain such heterogeneity. Indeed, a similar figure was found in Japan, with over 20 different variants now reported, although the people presented a relatively uniform genetic background as they had been living in isolated islands. ${ }^{9}$ In contrast, Val30Met is virtually the only mutation identified in Portuguese and Swedish patients. ${ }^{328}$ Its segregation with a unique haplotype in both countries has suggested the possibility of a common ancestor. ${ }^{29}$

Among the phenotypic characteristics, the most striking differences between the Portuguese and the French samples

Table 2 Estimations of penetrance in the French and Portuguese samples

\begin{tabular}{lllll}
\hline \multirow{4}{*}{$\begin{array}{l}\text { Age } \\
\text { (years) }\end{array}$} & \multicolumn{2}{l}{ Penetrance $(95 \%$ confidence interval) } & & \\
\cline { 2 - 5 } & French, all mutations & French, Val30Met & French, other mutations & Portuguese \\
\hline 30 & $0.02(0.01-0.02)$ & $0.01(0.01-0.02)$ & $0.02(0.01-0.02)$ & $0.26(0.22-0.29)$ \\
40 & $0.06(0.05-0.07)$ & $0.05(0.04-0.06)$ & $0.07(0.06-0.09)$ & $0.56(0.50-0.61)$ \\
50 & $0.18(0.15-0.21)$ & $0.14(0.10-0.17)$ & $0.22(0.17-0.26)$ & $0.80(0.75-0.85)$ \\
60 & $0.38(0.33-0.44)$ & $0.29(0.22-0.36)$ & $0.48(0.40-0.55)$ & $0.89(0.85-0.94)$ \\
70 & $0.64(0.58-0.71)$ & $0.50(0.40-0.60)$ & $0.77(0.69-0.85)$ & $0.91(0.86-0.95)$ \\
80 & $0.86(0.81-0.91)$ & $0.73(0.62-0.83)$ & $0.95(0.92-0.98)$ & $0.91(0.86-0.95)$ \\
\hline
\end{tabular}




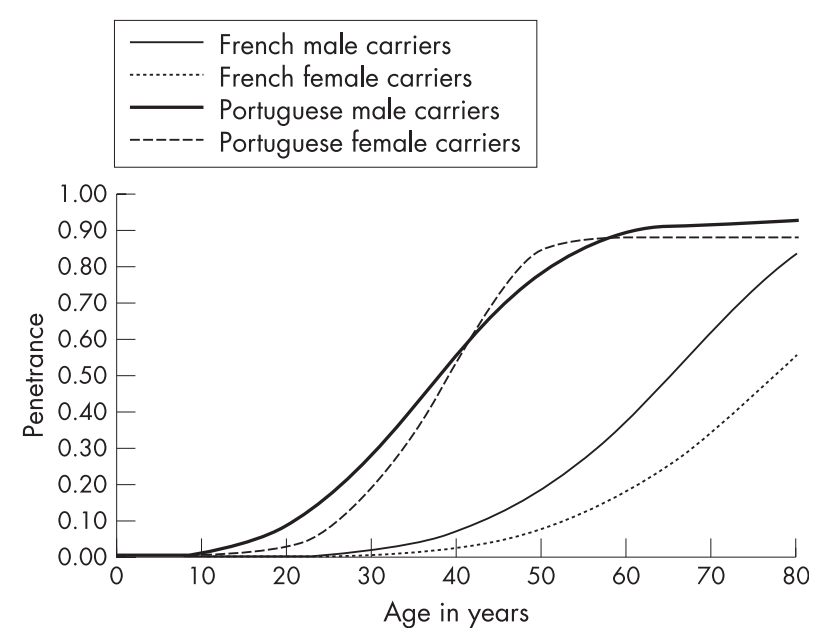

Figure 3 Estimated penetrance function for Val30Met carriers according to gender and geographic origin.

concerned age of onset. In the Portuguese families, a young age of onset was observed, with $75 \%$ of patients having their first symptoms before the age of 35 years. In this subgroup, more than $85 \%$ of the patients had a family history of TTR neuropathies, and the diagnosis was generally straightforward. These features are in line with the previous survey on TTR amyloid neuropathies in Portugal, although we did not find any difference in the mean age of onset between men and women. ${ }^{3} 30$ The age of onset was quite different in the French sample of TTR neuropathies, $85 \%$ of patients having their first symptoms over 50 years, with no difference according to gender. The Val30Met carriers and the patients carrying other variants of the TTR displayed similar characteristics, and virtually no genotype-phenotype correlation could be elicited. About half of the cases had no evidence of family history. A late mean age of onset (56 and 62 years respectively) and a frequent sporadic occurrence of the condition have been reported in Swedish families ${ }^{28}$ and in the Val30Met Japanese kindred described by Misu et al ${ }^{16}$ The high male:female ratio that we observed in the French index cases had been found in these two populations of late onset TTR amyloid neuropathy, but this feature has not received any explanation so far.

The major input of the present work was to provide unbiased estimates of penetrance and to test for a difference according to relevant factors: gender, genotype, and geographic origin. A previous report relied on segregation analysis only, without any molecular data. ${ }^{12}$ These authors concluded a penetrance estimate of $83 \%$, interestingly very close to that obtained in the French group in the present study. Other studies were based on the observation of unaffected carriers in families of cases. ${ }^{3}{ }^{13-16}$ In these studies, there was a tendency to conclude that late onset implied low penetrance. However, variable expressivity and incomplete penetrance are two different genetic parameters, even if they are sometimes correlated. ${ }^{31}$ In genetic diseases with variable age of onset, complete (or quasi-complete) penetrance implies that all carrier individuals will finally be affected if they live long enough. Therefore, it is not incompatible with rather late occurrence of the disease. Indeed, we found a much younger age of onset in the Portuguese group than in the French one, but the penetrance by the age of 80 years was only slightly higher in the Portuguese than in the French families $(91 \% \vee 86 \%)$, and not significantly different. By contrast, the penetrance values at intermediate ages display very high differences. For instance, the risk of disease at 50 years is only $18 \%$ in French carriers, but $80 \%$ in Portuguese carriers. This difference in penetrance cannot apparently be accounted for by a difference of genotype at the TTR gene. Indeed, the Val30Met French carriers display a lower penetrance at all ages than the carriers of the other mutations (penetrance by 80 years of age $73 \% v 95 \%$ ), but much lower than the Portuguese $(91 \%)$ who carry the same mutation. These differences remained highly significant even when allowing for a difference in ascertainment probabilities of patients between the two populations. At the present time, no explanation can be given for such a difference between French and Portuguese carriers of the Val30Met mutation; it could be caused either by differences in genetic background or by environmental differences between these two groups. Note that such differences not only exist between the populations, but also between families within the same population, as evidenced by the high degree of correlation within families for age of onset, which was found in both Portuguese and French families.

In accordance with the high sex ratio of 2.4 in favour in males found in the index cases, penetrance was found to be higher in males than females. However, the penetrance difference according to gender is rather small (and was evidenced only in Val30Met carriers), and cannot obviously account for the large excess of males among index patients. There is no argument for the existence of an ascertainment bias in favour of males and this difference remains to be explained.

It is important to mention that penetrance might be slightly overestimated because, in several pedigrees, we had to remove branches of the families where information on relatives was insufficient, or where the absence of other affected relatives among ancestors might have made suspect a possible de novo mutation. Indeed, some of these family branches with no affected relative might have been falsely attributed to de novo mutations, and their exclusion would tend to discard unaffected carriers, which would result in an overestimation of penetrance.

Another source of bias could be the extrapolation of age of onset from the age at death in some relatives whose age of onset was unknown, as the extrapolation was possible only for deceased individuals. We believe that this bias was

\begin{tabular}{|c|c|c|c|c|}
\hline \multirow{2}{*}{$\begin{array}{l}\text { Age } \\
\text { (years) }\end{array}$} & \multicolumn{4}{|c|}{ Penetrance $(95 \%$ confidence interval) } \\
\hline & French males & French females & Portuguese males & Portuguese females \\
\hline 30 & $0.02(0.01-0.02)$ & $0.01(0.00-0.01)$ & $0.30(0.25-0.35)$ & $0.21(0.17-0.25)$ \\
\hline 40 & $0.07(0.05-0.09)$ & $0.03(0.01-0.04)$ & $0.57(0.49-0.65)$ & $0.60(0.52-0.68)$ \\
\hline 50 & $0.19(0.13-0.24)$ & $0.08(0.04-0.11)$ & $0.79(0.71-0.87)$ & $0.86(0.77-0.95)$ \\
\hline 60 & $0.39(0.28-0.49)$ & $0.18(0.10-0.26)$ & $0.89(0.83-0.96)$ & $0.88(0.70-1.00)$ \\
\hline 70 & $0.64(0.51-0.76)$ & $0.35(0.22-0.48)$ & $0.92(0.84-1.00)$ & $0.88(0.48-1.00)$ \\
\hline 80 & $0.85(0.76-0.95)$ & $0.58(0.41-0.74)$ & $0.93(0.81-1.00$ & $0.88(0.10-1.00)$ \\
\hline
\end{tabular}


probably negligible as age of onset was found to be very similar both among individuals whose age of onset was extrapolated (40.3 (10.9)) and among individuals whose age of onset was accurately known (38.9 (14.9)). Addditionally, among affected relatives whose age of onset was known, no difference in age of onset was found between those living (38.9 (13.0) years) and those deceased (39.0 (15.5) years).

Overall, the present study allowed accurate estimates of penetrance in two European populations of TTR amyloid neuropathies and showed differences between these populations. In the future, it would be interesting to apply a similar approach to other families of various geographic origin, as the risk of developing the disease is likely to differ from one country to another. Such studies will contribute to a better understanding of the mechanisms underlying the disease expression. In addition, our results have direct clinical implications. They provide a useful tool to support genetic counselling of relatives before making a decision based on presymptomatic diagnosis. Indeed, the demand for predictive molecular testing has increased since liver transplantation has been performed more widely to treat this life threatening condition. The knowledge of the risk of being affected at various ages will also help substantially to improve the follow up of asymptomatic carriers and the management of patients at the very onset of symptoms. Finally, the incomplete penetrance documented in our study strengthens the present recommendation that liver transplantation should be performed only in symptomatic patients, at an early stage of the disease. ${ }^{11} 32$

\section{Authors' affiliations}

V Planté-Bordeneuve, A Ferreira, D Adams, G Said, Department of Neurology, Centre Hospitalier Universitaire de Bicêtre, Assistance Publique des Hôpitaux de Paris, France

J Carayol, F Clerget-Darpoux, C Bonaiiti-Pellié, Unité Inserm U535, Hôpital Paul-Brousse, Villejuif, France

M Misrahi, Laboratoire de Biologie cellulaire et moléculaire, Centre Hospitalier-Universitaire de Bicêtre, Assistance Publique des Hôpitaux de Paris, France

Correspondence to: Dr V Planté-Bordeneuve, Service de Neurologie, Centre Hospitalier Universitaire de Bicêtre, 94275 Le Kremlin Bicêtre, France; vplante@free.fr

\section{REFERENCES}

1 Said G, Ropert A, Faux N. Length-dependent degeneration of fibers in Portuguese amyloid polyneuropathy: A clinicopathological study. Neurology 1984:34:1025-32

2 Andrade C. A peculiar form of peripheral neuropathy: familial atypical generalized amyloidosis with special involvement of the peripheral nerves. Brain 1952:75:408-27.

3 Sousa A, Coelho T, Barros J, Sequeiros J. Genetic epidemiology of familial amyloidotic polyneuropathy (FAP)-Type I in Povoa do Varzim and Vila do Conde (North of Portugal). Am J Med Genet (Neuropsych Genet) 1995:60:512-21.

4 Araki S, Mawatari S, Ohta M, Nakajima A, Kuroiwa Y. Polyneuritic amyloidosis in a Japanese family. Arch Neurol 1968;18:593-602.

5 Andersson R. Hereditary amyloidosis with polyneuropathy. Acta Med Scand 1970; 188:85-94.

6 Reilly MM, Adams D, Booth DR, Davis MB, Said G, Laubriat-Bianchin M, Pepys MB, Thomas PK, Harding AE. Transthyretin gene analysis in European patients with suspected familial amyloid polyneuropathy. Brain $1995 \cdot 118: 849-56$
7 Benson MD. Leptomeningeal amyloid and variant transthyretins. Am J Pathol 1996;148:351-4

8 Saraiva MJM. Transthyretin mutations in hyperthroxinemia and amyloid diseases. Hum Mut 2001:17:493-503.

9 Ikeda S, Nakazato M, Ando Y, Sobue G. Familial transthyretin-type amyloid polyneuropathy in Japan. Clinical and genetic heterogeneity. Neurology 2002;58:1001-7

10 Planté-Bordeneuve V, Lalu T, Misrahi M, Reilly MM, Adams D, Lacroix C, Said G. Genotypic-phenotypic variations in a series of 65 patients with familial amyloid polyneuropathy. Neurology 1998;51:708-14.

11 Adams D, Samuel D, Goulon-Goeau C, Nakazato M, Costa PM, Feray C, Planté V, Ducot B, Ichai P, Lacroix C, Metral S, Bismuth H, Said G. The course and prognosis factors of familial amyloid polyneuropathy after liver transplantation. Brain 2000;123:1455-1504.

12 Sakoda S, Suzuki T, Higa S, Ueji M, Kishimoto S, Hayashi A, Yasuda N, Takaba Y, Nakajima A. Genetic studies of familial amyloid polyneuropathy in the Arao district of Japan. I. The genealogical survey. Clin Gen 1983;24:334-8.

13 Drugge U, Andersson R, Chizari F, Danielsson M, Holmgren G, Sandgren O, Sousa A. Familial amyloidotic polyneuropathy in Sweden: a pedigree analysis. J Med Genet 1993;30:388-92.

14 Coelho T, Sousa A, Lourenco E, Ramalheira J. A study of 159 Portuguese patients with familial amyloidotic polyneuropathy (FAP) whose parents were both unaffected. J Med Genet 1994:31:293-9.

15 Holmgren G, Costa PM, Andersson C, Asplund K, Steen L, Beckman L, Nylander PO, Teixeira A, Saraiva MJ, Costa PP. Geographical distribution of TTR met 30 carriers in northern Sweden: discrepancy between carrier frequency and prevalence rate. J Med Genet 1994;31:351-4.

16 Misu K, Hattori N, Nagamatsu M, Ikeda S, Ando Y, Nakazato M, Takei Y, Hanyu N, Usui Y, Tanaka F, Harada T, Inukai A, Hashizume Y, Sobue G. Lateonset familial amyloid polyneuropathy type I (transthyretin Met30-associated familial amyloid polyneuropathy) unrelated to endemic focus in Japan. Clinicopathological and genetic features. Brain 1999:122:1951-62.

17 Izumoto S, Younger D, Hays AP, Martone RL, Smith RT, Herbert J. Familial amyloidotic polyneuropathy presenting with carpal tunnel syndrome and a new transthyretin mutation, asparagine 70. Neurology 1992:42:2094-102.

18 Le Bihan C, Bonaiiti-Pellié C. A method for estimating cancer risk in p53 mutation carriers. Cancer Detection Prev 1994:18:171-8.

19 Le Bihan C, Moutou C, Brugieres L, Feunteun J, Bonaiti-Pellie C. ARCAD: A method for estimating age-dependent disease risk associated with mutation carrier status from family data. Genet Epidemiol 1995;12:13-25.

20 Chompret A Brugieres L, Ronsin M, Gardes M, Dessarps-Freichey F, Abel A Hua D, Ligot L, Dondon MG, Bressac-de-Paillerets B, Frebourg T, Lemerle J, Bonaiti-Pellie C, Feunteun J. p53 germline mutations in childhood cancers and cancer risk for carrier individuals. Br J Cancer 2000;82:1932-7.

21 Sposto R. Cure model analysis in cancer: an application to data from the Children's Cancer Group. Stat Med 2002;21:293-312.

22 Elston RC, Stewart J. A general model for the genetic analysis of pedigree data. Hum Hered 1971;21:523-42.

23 Morton NE. Genetic tests under incomplete ascertainment. Am J Hum Genet 1959:11:1-16.

24 Lalouel JM. GEMINI-a computer program for optimization of general non linear functions. Technical report no 14. Salt Lake City: University of Utah, Department of Medical Biophysics and Computing, 1979.

25 Brookmeyer $\mathbf{R}$, Crowley J. A confidence interval for the median survival time. Biometrics 1982:38:29-41.

26 Karrison T. Confidence intervals for median survival times under piecewise exponential model with proportional hazards covariate effects. Stat Med 1996; 15:171-82

27 Misrahi M, Planté V, Lalu T, Serre L, Adams D, Lacroix DC, Said G. New transthyretin variants SER91 and SER 116 associated with familial amyloid polyneuropathy. Hum Mut 1998;12:71.

28 Sousa A, Andersson R, Drugge U, Holmgren G, Sandgren O. Familial amyloidotic polyneuropathy in Sweden: geographical distribution, age of onset, and prevalence. Hum Hered 1993:43:288-94.

29 Almeida MR, Aoyama-Oishi N, Sakaki Y, Holmgren G, Ulf D, Ferlini A, Salvi F, Munar-Qués M, Benson MD, Skinner M, Costa PP, Saraiva MJ. Haplotype analysis of common transthyretin mutations. Hum Genet 1995:96:350-34

30 Sequeiros J, Sousa A, Coelho T. Sex difference and age-dependent penetrance in FAP-type I. In: Natvig JB, Forre O, Husby G, Husebekk A Skogen B, Sletten K, Westermark P, eds. Amyloid and amyloidosis. Dordrecht: Kluver, 1990:687-690.

31 Bonaïti-Pellié C, Clerget-Darpoux F, Babron MC. Hereditary retinoblastoma: can balanced insertion entirely explain the differences of expressivity among families? Hum Genet 1990;86:203-8.

32 Suhr OB, Herlenius G, Friman S, Ericzon BG. Liver transplantation for hereditary transthyretin amyloidosis. Liver Transplant 2000;6:263-76. 